\title{
Prueba de inclinación en la evaluación del síncope o presíncope de etiología no precisada en niños y adolescentes
}

\author{
Eduardo Dumas R. '; Francisco J. Radrigán V. ${ }^{\text {; }}$ Pilar Amaiz G. '; \\ Felipe Heusser R.' ; Gastón Chamorro S. ${ }^{1}$; Susana Becerra G. ${ }^{2}$; \\ Susy Cohen C. ${ }^{2}$; Jorge E. Jalil M. ${ }^{1}$

\begin{abstract}
Head-up tilt test in the clinical evaluation of unexplained syncope or presyncope in children and adolescents
\end{abstract}

\begin{abstract}
Unexplained syncope or presyncope are rather common problems in children, whose evaluation is not simple. In adults, the hilt lest alows to reproduce sympioms and some ossociated hemodynamic mechonisms in 30 to $75 \%$ of the patients with this condition. The purpose of this wa:k has been to analyze hemodynamic responses during $70^{\circ}$ rilt test in 26 consecutive polients younger than 19 years, releced to our center with the diagnosis of unexploined syncope in a period of 2 years. Fithy percent of the tilt lest were positive, most of them using isoproterenol (meon dose \pm 0.4 micrograms/minulet, increasing heart rale by $41 \%$. Syslotic arterial pressure decreased significantly in all patients with a positive tilt test to less than $70 \mathrm{mmHg}$. Reduction of heart rate was also observed, rarely to marked bradycardia by the end of the rest. Original symptoms were always reproduced during the lest. It is concluded that ti 1 test may constitute an imoorlont lest in the clinical workup of unexpkoined syncope in children and adolescents. This method reproduces clinical symptors associated ic syncope or presyncope and precises their hemodynamic mechonisms in aporoxima y $50 \%$ of rese patients.
\end{abstract}

[Koy words. syncope, tilt tesl, isoprotererol.]

El síncope recurrente y el presícope son problemas relativamente frecuentes en niños. En adultos, aproximadamente en la mitad de los casos de síncope o presíncope no es posible determinar la causa pese a una exhaustiva investigacion $n^{1 \cdot 3}$. La experiencia en estos pacientes, con síncope o presíncope de etiología no precisada (SENP) y sin evidencias de cardiopatía es que la prueba de inclinación permite reproducir el síntoma y algunos de sus mecanismos hemodinámicos asociados en 30 a $75 \%$ de los $\operatorname{casos}^{1-5}$. En una serie anterior la sensibilidad global ha sido de $45 \%$ en pacientes con SENP de todas las edades ${ }^{6}$.

El fundamento fisiológico de este examen es que el síncope vasovagal o vasodepresor serfa

I. Pontificia Universidad Catolica de Chile, DeparIamentos de Enfermedades Cardiovasculares y Pediatría.

2. Enfermera Universitaria, Pontificia Unversidad Católica de Chile. Departamentos de Enfermedades Cardiovasculares y Pediatría una exageración del reflejo de Bezold-Jarisch, que normalmente se puede desencadenar al estimular mecanorreceptores localizados en el ventrículo izquierdo. Estos receptores corresponderían a terminaciones vagales denominadas fibras $\mathrm{C}^{1.7}$ que son excitadas por catecolaminas, el sistema nervioso simpático y el aumento de la presion intraventricular izquierda. Esta respuesta sería acentuada en algunos sujetos por la inclinación en 70 grados, que además reduce el retorno venoso y produce una respuesta simpática compensatoria. El isoproterenol es una catecolamina sintética, aumenta el gasto cardíaco y reduce el volumen ventricular izquierdo. Experimentos con animales y datos clínicos en humanos han mostrado que la reducción del tamaño ventricular izquierdo con obliteración de] apex crearía una gradiente que estimula a estas fibras $C^{8.9}$. Como consecuencia aumenta la actividad vagal eferente y disminuye la actividad simpática, causando hipotensión arterial o bradicardia y pérdida de conciencia secundaria a hipoperfusión cerebral. 
No existen muchos estudios sobre la prueba de inclinación en niños y adolescentes, reportándose sensibilidades de $43 \%$ y $80 \%$ en dos series clínicas ${ }^{10-12}$. El objetivo de este trabajo fue describir las respuestas hemodinámicas durante la prueba de inclinación en todos los pacientes menores de 19 años de edad, referidos a nuestro centro por síncope de causa no precisada durante dos años.

\section{Pacientes y Métodos}

Entre enero de 1992 y enero de 1994, 26 pacientes consecutivos menores de 19 años de edad con diagnóstico de SENP fueron referidos a nuestro laboratorio. A todos ellos se les realizó prueba de inclinación como parte de su esiudio. Se consideró síncope (n: 17) o presíncope (n: 9) a la perdida total o parcial de conciencia respectivamente, con incapacidad para mantener el Iono postural debido a hipoperfusion cerebral ${ }^{13-15}$.

El diagnóstico de SENP se hizo en pacientes con síncope en quienes el electrocardiograma de superficie. registro Holter de 24 horas, ecocardiograma bidimensional y con Doppler y evaluación neurológica con electroencefalograina no explicaban el cuadro clínico. Se incluyó un paciente con enfermedad del nódulo sinusal y otro con doble nódulo atrioventricular, pues en sus registros Holter no se encontraron razones para el síncope. La edad de los 26 pacientes (13 mujeres) descritos era 14,6 $\pm 0,61$ (mediana 15,5; márgenes ? a 18) años; peso $54 \pm 2,2 \mathrm{~kg}$, talla $159,4 \pm 2,5 \mathrm{~cm}$ Siete pacientes habían sufrido uno o dos episodios de síncope, doce registraban 3 a 5 episodios y siete babian sufrido 60 más crisis

Se suspendió todo fármaco 24 horas previas al examen, e) que se efectú en una sala tranquila con fuz natural, a media tarde, después de un almuerzo liviano. En todos los pacientes, previo al examen, se realizó un electrocardiograma de 12 derivaciones. Se monitoriz6 la sefial electrocardiogrifica en forma continua con equipo Quinton, Instrumed Co. 4000 y la presión arterial sistólica, diastó. lica y media minuto a minuto con un equipo Critikon Dynamap 1846 SX/P version 089.

El protocolo utilizado consistio en cuatro etapas y se realizó en una mesa para inclinación dotada de base para apoyo de pies, corteas de seguridad y un motor electrico que permite modificar su ángulo con respecto a la horizontal de manera rípida y graduada. En la primera etapa el paciente es colocado en decúbito dorsal en ángulo de $0^{\circ}$ sobre la horizontal durante 5 minucos y comesponde a la condición basal. Luego se pasa a la segunda etapa, sin drogas, inclinando al paciente en $70^{\circ}$, la cabeza arriba, para mantenterle en esta pusición por 20 minutos (o menos si el examen es positivo). Si el examen es negativo, se continúa con la tercera etapa, a $\mathbf{0}$ grados ahora con isoproterenol en dosis de 0,25 a 1 hg por minuto IV mediante bomba de infusión continua, aumentándolo progresivamente con el objeto de lograr frecuencias cardíacas (FC) mayores -en $30 \%$ - que la basal. Una vez lograda la frecuencia deseada se mantiene esa dosis por $\mathbf{5}$ minutos y se pasa a la etapa cuatro inclinando la camilla a 70 grados, manteniendo la administración de isoproterenol durante otros 20 minutos o antes de dicho plazo si el examen es positivo. A contar de enero de 1994, y al comprobar mayor sensibilidad y simplicidad del protocolo con isoproterenol exclusivamente, se cambio a un protocolo acortado, eliminandose la etapa dos previamente descrita $\left(70^{\circ} \mathrm{sin}\right.$ isoproterenol). Los criterios de positividad del examen fueros: hipotensión arterial (presión sistólica menor de $85 \mathrm{mmHg}$ ) acompanada de fatiga, colapso inminente (presíncope) o pérdida de conciencia (síncope): bradicardia sinusal o ritmo nodal sintomaticos de $<40$ por minuto: bloqueo auriculoventricular de $2^{\circ}$ grado de tipo vagotónico o con secuencias de Wenckebach: síncope vaso-depresor maligno, definido como sincope acompañado de asistolía prolongada ( $>8$ segundos). Cuando estas manifestaciones ocurrieron se dio término al examen, volviendo al paciente a la horizontal ( 0 grados) e interrumpiendo la infusión de isoproterenol.

Los resultados son presentados como promedios y error estándar. Ocasionalmente se señala el rango y el intervalo de confianza de la diferencia con un nivel de significancia de $955 \%(95 \%$ IC). Se utilizó petieba de $t$ de Student para muestras independientes, ANOVA de un factor y ANOVA para pruebas repetidas con prueba de Tukey para comparaciones múltiples.

\section{Resultados}

Se realizaron 26 procedimientos. En la mitad de los pacientes (n: 13) se registró una respuesta positiva, que reprodujo los síntomas por los que consultaron (tabla 1).

En $21 / 26$ pacientes se realizó la prueba de inclinación con las cuatro etapas y $47,6 \%$ (10/ 21) resultados positivos. En los otros cinco casos se hizo la prueba acortada en tres etapas (eliminando la fase de $70^{\circ} \mathrm{sin}$ isoproterenol) con resultado positivo en $3 / 5$. En tres pacientes la prueba resultó positiva sin isoproterenol (grupo A), pero en la mayoría de los pacientes $(10 / 13,77 \%)$ la prueba resultó positiva con suministro de isoproterenol (grupo $\mathrm{B}$ ).

En los pacientes cuya prueba fue positiva sin isoproterenol el tiempo transcurrido hasta obtener dicho resultado fue $12 \pm 3,6$ (márgenes 7 a 19) minutos. En los casos cuya prueba fue positiva bajo isoproterenol la respuesta se registró al cabo de 13,1 $\pm 2,2$ (márgenes 2 a 22) minutos (ns).

La dosis de isoproterenol requerida para obtener la respuesta en los pacientes con prueba positiva fue $3,1 \pm 0,41$ (márgenes 2 a 6 ) $\mu \mathrm{g}$ por minuto, lográndose incrementos promedio de $41 \%$ de la frecuencia cardíaca basal. En las pruebas negativas la dosis promedio fue $1,88 \pm$ 
Tabla 1

Características de los pacientes con una prueba de inclinación positiva (n: 13/26)

\begin{tabular}{|c|c|c|c|c|c|c|}
\hline Grupo & Edad & Sexo & Antecedentes & Bolter & $\begin{array}{c}\text { Tiempo } \\
\text { (min) }\end{array}$ & Ritmo \\
\hline \multirow{8}{*}{ A } & 18 & $\mathbf{M}$ & - & Normal & 19 & BS \\
\hline & 14 & $\mathrm{~F}$ & - & Normal & 7 & BS \\
\hline & 11 & $\mathbf{M}$ & Epilepsia (1) & Normal & 10 & $\mathrm{BS}, \mathrm{N}, \mathrm{AS}$ \\
\hline & 13 & $\mathbf{F}$ & - & Normal & 5 & BS \\
\hline & 16 & M & (2) & ENS & 20 & BS \\
\hline & 15 & $\mathrm{~F}$ & - & Normal & 14 & Nodal \\
\hline & 17 & $\mathrm{~F}$ & (3) & NAVD & 12 & Nodal \\
\hline & 17 & $\mathrm{~F}$ & - & Normal & 18 & $B S, W$ \\
\hline \multirow[t]{5}{*}{ B } & 11 & $\mathrm{~F}$ & - & Normal & 20 & Nodal \\
\hline & 17 & $\mathrm{~F}$ & HIP & Normal & 2 & BS \\
\hline & 16 & $\mathrm{~F}$ & - & Normal & 16 & BS \\
\hline & 1.3 & $\mathrm{~F}$ & - & Normal & 5 & Nodal \\
\hline & 18 & $M$ & - & Normal & 17 & Nodal \\
\hline
\end{tabular}

Grupo A: Prueba positiva sin isoproterenol; Grupo B: preba positiva con isoproterenol; M: masculino: F: femenino; Tiempo: de respuesta desde el inicio de la etapa a $70^{\circ}$ (min); BS: bradicardia sinusal absoluta o relativa; $\mathrm{N}$ : ritmo nodal; AS: asistolía; W: bloqueo A.V $2^{a}$ grado tipo Wenckebach; ENS: enfermedad del nódulo sinusal; HIP: hemibloqueo izquietdo posterior; NAVD: nódulo A-V doble.

(1) Tratado con fenobarbical que se suspendió tres días antes del examen. Prueba positiva sin isoproterenol, asjstolía prolongada, requirio masaje cardíaco externo. (2) Holter con signos de ENS. Bradicardia sinusal hasta 25 por min y pausas hasta 2,7 seg. Ergomerría sin insuficiencia cronotrópica. (3) Holter y estudio electrofisıológico: nódulo A-V doble. No se indujo taquicardia. Elecirocardiograma basal, de esfuerzo y ecocardiograma fueron norinales.

$0,29 \mu \mathrm{g}$ por minuto (márgenes 0,5 a 4 ) con incremento promedio de la frecuencia cardíaca de $33,2 \%$ (ns comparado con el aumento de la frecuencia en los casos positivos). La dosis de isoproterenol fue significativamente mayor en los pacientes que cuya prueba de inclinación fue positiva ( $p<0,02$; IC $95 \%$ de 0,22 a 2,26$)$.

En todos los casos cuyo examen dio resultado positivo, la hipotensión arterial y los trastornos del ritmo desaparecieron rápidamente al colocat al paciente en posición horizontal y suspender la infusión de isoproterenol. En un niño de 11 años de edad, con síncope vasodepresor maligno, la asistolía duró aproximadamente 12 segundos, requirio masaje cardíaco externo, recuperándose rápidamente y sin problemas. Un paciente sufrío arritmias inducidas por isoproterenol (extrasístoles auriculares aislados y en salvas y extrasistoles ventriculares aislados) que no estaban presentes antes o después del examen.

En los pacientes con una prueba positiva $\sin$ isoproterenol (grupo $A, n=3$ ) se registró un descenso significativo de la presión arterial sist6́lica, desde $108,5 \pm 2$ a $68,3 \pm 7,3 \mathrm{mmHg}$ (p<0,01) y de la presión arterial media, desde $82 \pm 0,1$ a $56 \pm 6,7 \mathrm{mmHg}(\mathrm{p}<0,02)$, sin cambios significativos de la presion arterial diastólica y la frecuencia cardíaca. En los casos positivos con isoproterenol (grupo $B, n=10$ ) se registró un descenso significativo de las presiones arterial sistólica, diastolica y media al momento de hacerse positivo el examen. La frecuencia cardíaca aumentó en $41 \%$, respecto a la basal en posición horizontal, con el uso de isoproterenol, para luego disminuir en forma significativa al momento de hacerse positivo el examen (tabla 2). En general, la hipotensión arterial tendía a preceder a la disminución de la frecuencia cardíaca.

De los casos positivos sin isoproterenol dos tuvieron bradicardia sinusal de 40 y 56 por min y uno con ritmo nodal a 73 por min al finalizar el examen. En los casos positivos con isoproterenol se document 6 bradicardia sinusal relativa en $4 / 10$ casos y ritmo nodal en $6 / 10$ casos al término del examen. La respuesta del ritmo no mostró diferencias en los casos positivos con o 
Tabla 2

Respuestas bemodinámicas a la pnueba de inclinación en los casos positivos con isoproterenol (n: 10)

\begin{tabular}{lcrc}
\hline & Busal $\left(0^{\circ} \sin\right.$ ISO) & $0^{\circ}$ con ISO & Final $\left(70^{\circ}\right.$ con ISO) \\
\hline PAS & $118,4 \pm 5,5$ & $126.5 \pm 4,2$ & $67,7 \pm 3,7^{* \#}$ \\
PAD & $61,9 \pm 2,8$ & $56,0 \pm 2,7$ & $39,5 \pm 2,3^{*} \#$ \\
PAM & $84,1 \pm 3,5$ & $77,3 \pm 3,5$ & $49,6 \pm 3,1^{* \#}$ \\
FC & $78,2 \pm 6,9$ & $110,6 \pm 8,6^{*}$ & $68,8 \pm 3,2 \#$
\end{tabular}

PAS: presión arterial sistolica (mmHg). PAD: presión arterial diastólica $(\mathrm{mmHg})$. PAM: presión arterial media $(\mathrm{mmHg})$; FC: frecuencia cardíaca por min. ISO: isoproterenol.

${ }^{*}: \mathrm{p}<0,05$ vs BASAL; ; $\mathrm{p}<0,05$ vs $0^{\circ}$ con isoproterenol.

sin isoproterenol. Un paciente del grupo A presentó rítmo nodal seguido de bloqueo auriculoventricular de segundo grado de tipo vagotónico y también de segundo grado de Wenckebach. Todos los trastornos del ritmo revirtieron en forma espontánea al terminar el examen.

\section{Comentario}

Los resultados en esta serie en niños y adolescentes son similares a los encontrados en la literatura extanjera y a nuestros resultados previos en relación a la población total de pacientes sometidos a prueba de inclinación. La sensibilidad global de esta serie fue $50 \%$. Los dos trabajos en niños $\mathrm{y}$ adolescentes previamente mencionados reportan sensibilidades de 43 y $80 \%$ respectiyamente ${ }^{10 .}{ }^{12}$. Sin embargo, por varias razones dichas cifras no son del todo comparables a nuestros resultados. Los protocolos utilizados son diferentes en cuanto a duración de las fases, grados de inclinación y uso de isoproterenol. En uno de ellos ${ }^{10}$ se utilizó prueba sin isoproterenol, una etapa basal de 10 min e inclinación de $60 \%$ por una hora. La positividad se observó en promedio a los $48 \mathrm{~min}$ del procedimiento en $43 \%$ de los casos. Recientemente se ha comunicado que la prueba de inclinación bajo isoproterenol tiene un rendimiento diagnóstico bastante superior a la prueba prolongada de 45 min sin isoproterenol a inclinacion $70 \%$ en pacientes con síncope neurocardiogénico ${ }^{16.18}$, lo que sugiere que sería más conveniente realizar la prueba exclusivamente con el medica- mento, acortando sustancialmente su duración y simplificando el examen, tal como lo estamos haciendo en la actualidad. Por otro lado, con un protocolo similar al nuestro, tiempos ligeramente diferentes e inclinación de $60 \%$, se ha registrado una sensibilidad de $80 \%$, si bien las frecuencias cardíacas obtenidas con isoproterenol -en el aludido trabajo- alcanzaron a $50 \circ 60 \%$ sobre los valores basales, lo que podría limitar la especificidad del examen en ese caso ${ }^{12}$.

Con la prueba de inclinación se pueden inducir respuestas positivas en 8 a $31 \%$ de los sujetos normales. Los factores involucrados en la obtención de tal respuesta son grado de inclinación, frecuencia ventricular alcanzada con el isoproterenol y el tiempo de duración del examen ${ }^{18}$.

Se ha cuestionado la prueba de inclinacion con isoproterenol como un examen especifico de síncope vasodepresor, pero con experiencias en que se utilizo $80 \%$ de inclinación. la que ha demostrado inducir falsos positivos ${ }^{3}$. En 45 sujetos normales sometidos a prueba de $60^{\circ}, 70^{\circ}$ y $80^{\circ}$ de inclinacion con isoproterenol, la frecuencia cardíaca aumentó en s6lo $20 \%$ y se registraron $27 \%$ de falsos positivos con $80 \%$ de angulación, mientras en $60^{\circ}$ y $70^{\circ}$ con isoproterenol la especificidad fue 93 y $85 \%$, respectivamente, sin diferencias con la especificidad del examen en ausencia de isoproterenol en los mismos grados de inclinación ${ }^{18}$. En un trabajo previo ${ }^{6}$, observamos que los pacientes que durante la prueba presentaron reaccion vasodepresora tendian a ser menos sensibles al efecto cronótropo positivo del isoproterenol, ya que para lograr incrementos relativos similares de frecuencia cardíaca necesitaban prácticamente un $\mu \mathrm{g} /$ min mâs que los pacientes con reacción vasodepresora negativa. Esta observación contrastaba con las experiencias antes mencionadas ${ }^{3,4} \mathrm{y}$ pensábamos que la diferencia estaba dada por la pequeña proporción de pacientes de menor edad de nuestra primera serie; sin embargo, los resultados de este trabajo confirman nuestra primera impresión de menor sensibilidad a la taquicardia inducida por el isoproterenol en estos pacientes y también en personas jóvenes, fenómeno que será necesario caracterizar mejor.

En los últimos 10 minutos de la prueba, en los pacientes con respuesta positiva, la hipotensión arterial fue en general previa a las manifestaciones electrocardiográficas y al presíncope o 
síncope. Esta información reafirma que el elemento primordial de este perfil temporal es la hipotensión arterial y que la disminución de la frecuencia ventricular, si bien fue significativa, rara vez fue acentuada. De hecho, salvo por el paciente que sufrió asistolía prolongada, la menor frecuencia cardíaca observada en esta serie de niños y adolescentes fue de 40 latidos por minuto. Previamente a esta secuencia se observa taquicardia sinusal secundaria a estimulación adrenérgica ${ }^{19}$, lo que constituye precisamente el rbjetivo de emplear isoproterenol y que desencadenaría el reflejo de Bezold-Jarish en forma pronunciada. La respuesta hemodinámica positiva a la prueba de inclinación con isoproterenol consistió en una caída significativa de la presión arterial sistolica, diastólica y media y de la frecuencia ventricular. El número de sujetos con respuesta positiva sin isoproterenol fue tan pequeño que no permite generalizaciones en ese subgrupo de pacientes.

Estos datos concuerdan con la concepción fisiopatologica del síncope vasodepresor, en que se requiere hiperestimulación adrenérgica para desencadenar una reacción vagal dependiente de estimulación de las fibras $\mathrm{C}$ del ventrículo izquierdo. Nuestros datos no explican cuál es la característica que hace que algunos sujetos reaccionen de una forma más pronunciada con hipotensión arterial y síntomas, frente al mismo estímulo. Una alternativa a la prueba de inclinación para niños que sufren de síncope o presíncope de etiología no precisada con corazón normal es la maniobra ortostática, en que el niño es puesto de pie en $90^{\circ}$, sin emplear mesa inclinable ni medicamentos ${ }^{20}$, pero no conocemos su rendimiento en comparación con la prueba que se comenta ni tenemos experiencia con ella.

La prueba de inclinacion puede constituir un examen importante en el estudio del síncope de etiología no precisada en niños y adolescentes, al permitir reproducir la sintomatología particular en cada caso de síncope o presíncope y precisar los mecanismos hemodinámicos participantes en aproximadamente $50 \%$ de estos pacientes.

\section{Resumen}

El síncope y el presíncope recurtentes y de etiología no precisada son problemas relativa- mente frecuentes en niños y, en general, de dificil evaluación. En adultos, la prueba de inclinación permite reproducir los síntomas y algunos de los mecanismos hemodinámicos asociados en 30 a $75 \%$ de los casos. Se describen las respuestas hemodinámicas durante la prueba de inclinación a $70^{\circ}$ en 26 pacientes consecutivos menores de 19 años de edad, referidos a nuestro centro por dicho problema en un período de dos años. Se obtuvieron respuestas positivas en $\mathbf{5 0 \%}$ de los casos, en la mayoría de ellos utilizando isoproterenol (dosis promedio $3,1 \pm 0,4 \mu \mathrm{g} / \mathrm{min}$ ), con incremento promedio de la frecuencia cardíaca de $41 \%$. La presion arterial sistólica disminuy6 significativamente, hasta menos de 70 $\mathrm{mmHg}$, en todos los pacientes con respuesta positiva. También disminuyo la frecuencia cardíaca, lo que rara vez fue acentuado, al momento de hacerse positivo el examen. La hipotensión arterial fue simultánea o precedio a la bradicardia. En todos los casos positivos se reprodujeron los síntomas que motivaron la consulta. Esta prueba puede ser de importancia en el estudio diagnóstico del síncope de causa no precisada en niños y adolescentes y, al reproducir los síntomas asociados, permite precisar los mecanismos bemodinámicos participantes en aproximadamente el $50 \%$ de estos pacientes.

(Palabras clave: síncope, prueba de inclinación, isoproterenol.)

\section{Referencias}

I. Kenny RA, Ingram A, Bayliss J, Sutton R: Head-Up Tilt: a useful test for investigating unexplained syncope. Lancet 1986; 1: 1352-1355.

2. Waxman M, Yao L, Cameron D, Wald $R$, Roseman J: Isoproterenol induction of yasodepressor-type reaction in vasodepressor-prone persons. Am J Cardiol 1989; 63: $58-65$.

3. Kapoor W, Barnt N: Evaluation of syncope by upright tilt testing with isoproterenol. A nonspecific test. Ann Int Med 1992; 116: 358-363.

4. Fitzpatrick AP, Theodorakis $G$, Vardas $P$, Sutton $R$ : Methodology of head-up tilt testing in patients with unexplained syncope. JACC 1991; 17: 125-130.

5. Grubb BP, Temesy-Amos P. Hahn $H$, Elliot L: Utility of upright tilt-table testing in the evaluation and management of syncope of unknown origin. Am $J$ Med 1991; 90:6-10

6. Jalil $f$, Radrigún F, González $R$, Godoy 1 , Fajuri A, Chamorro G. Casanegra P, Valenzuela $C$ : Prueba de Inclinación (Tilt Test): Respuestas bemodinámicas en paciedtes con síncope o presíncope de etiologia no precisada. Rev Med Chile 1993; 1367-1373. 
7. Almquist A, Goldenberg 1, Milstein $S$ et al: Provocation of bodicardia and hypotension by isoproterenol and upright posture in paijents with unexplained syacope. N. Engl J Med 1989; 320: 346-35I.

8. Oberg 8 , Thoren $P$ : Increased activity in left ventricular receptors during hemorrhage or occlusion of caval veins in the cat. A possible cause of the vasovagal reaction. Acta Physiol Scand 1972; 85: 164-173.

9. Marck AL: The Bezold-Jarish reflex revisited: clinical implications of inhibitory reflexes originating in the heart. JACC 1983; 1; 90-102.

10. Lerman-Sagie T, Rechavia E, Strasberg B. Sagie A, Blieden L: Head-Up Tilt for the evaluation of syncope of unknown origin in children. J Pediatr 1991; 118: 676-679.

11. Hannen D. Ross B: Head-up tilt testing in children who faint. J Pediatr 1991: 118: 731-732.

12. Thilenius $O$. Qwinones J, Tarek H. Novak J: Tilt test for diagnosis of unexplained syncope in pediatric patients. Pediatrics 1991; 87: 334-338.

13. Kapoor W. Karpf $M$, Wieand S, Peterson JA: Prospective evaluation and follow-up of patients with syncope. N Engl J Med 1983; 309: 197-204.
14. Kapoor W: Evaluation and oulcome of patients with syocope. Medicine 1990; 69: 160.175.

15. Kapoor W: Diagnostic evaluation of syncope. Am J Med 1991: 90 : 91.105.

16. Jaiji J. Radrigón F. González R, Chamorro G, Dumas E. Léniz P, Lopez $G$ : Comparacion de tilt test con isoproterenol versus tilt test prolongado en pacientes con síncope neurocardiogénico. Rev Chil Cardiol 1994; 13: $4-7$.

17. Sheldon $R$ : Evaluation of a single-stage isoproterenol. tilt table test in patients with syncope. JACC 1993; 22; I14-118.

18. Natale A, Sra J, Avilall B, Dhala A, Sanjay D. Jazayeri $M$. Wase A. Sheth $M$, Athtar $M$ : Specifity ofi bead-up tilt: effects of degree of tilt arid isoproterenol infusion. JACC 1993;21: 157A

19. Sra JS, Jazayeri MJ. Murthy VS et al: Sequential cathecolamine changes during upright tilt: possible, hotmonal mechanisms responsible for pathogene'sis of neusocardjogenic syncope. JACC 1991: 17: 2 I A.

20. Baiaji $S$, Oslizlok $P$, Allen M. McKay C Gillerfe $P$, Neurocardiogenic syncope in children with a ncirmal beart. JACC 1994; 23: 779-785.

Esta publicación está disponible en copias de microfilms de 16 y $35 \mathrm{~mm}$ y microfichas de $105 \mathrm{~mm}$, las que pueden solicitarse a:

University Microfilms International

300 North Zeeb Road

Ann Arbor, Michigan 48106, USA.

This journal is also available in $16 \mathrm{~mm}$ microfilm, $35 \mathrm{~mm}$ microfilm and 105 mm microfilm copies through

University Microfilms International,

300 North Zeeb Road.

Ann Arbor, Michigan 48106, USA. 